\title{
High vancomycin serum trough is not associated with reduction of mortality in methicillin-resistant Staphylococcus aureus bloodstream infections
}

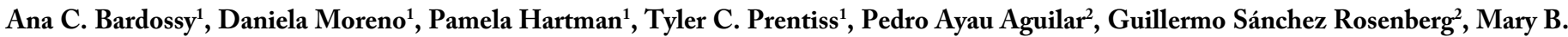 \\ Perri $^{1}$, Khulood Rizvi ${ }^{1}$, Tooba Rehman ${ }^{1}$, Ayesha Niazy ${ }^{1}$, Meredith Mahan ${ }^{1}$, Geehan Suleyman ${ }^{1}$, Vanthida Huang ${ }^{3}$ Katherine Reyes ${ }^{1}$ and \\ Marcus J. Zervos ${ }^{1,4 *}$
}

${ }^{1}$ Henry Ford Health System, Detroit, MI, USA

${ }^{2}$ Universidad Francisco Marroquin, Guatemala, Guatemala

${ }^{3}$ Midwestern University College of Pharmacy-Glendale, Glendale, AZ, USA

${ }^{4}$ Wayne State University School of Medicine, Detroit, MI, USA

\begin{abstract}
The current Infectious Diseases Society of America (IDSA) and the American Society of Health-System Pharmacists (ASHP) guidelines recommend a vancomycin serum trough concentration of 15 to $20 \mathrm{mg} / \mathrm{L}$ in patients with methicillin-resistant Staphylococcus aureus (MRSA) bloodstream infection (BSI). The objective of this study was to evaluate the mortality difference in MRSA BSI pre and post hospital-wide implementation of higher serum trough concentration per IDSA/ ASHP guidelines. This was a retrospective cohort study performed in an integrated hospital health system (2238 beds) in Southeast Michigan. We evaluated 1173 consecutive individual patients with MRSA BSI over a 9-year period. The vancomycin minimum inhibitory concentrations (MICs) were determined by Etest method for all isolates. Attainment of vancomycin serum trough concentration per IDSA/ASHP guidelines was implemented in January 2010 by clinical pharmacist as part of the antimicrobial stewardship program. During the study period, the mean vancomycin MIC was $1.57 \pm 0.26 \mathrm{mg} / \mathrm{L}$, the percentage of MRSA isolates with vancomycin $\mathrm{MIC} \geq 2 \mathrm{mg} / \mathrm{L}$ was $17.5 \%$, and the 30 -day all-cause mortality was $16.5 \%$. There was no difference in mortality during the 9 -year period ( $\mathrm{p}=0.193$ ). There was no change in all-cause mortality for MRSA BSI after the hospital-wide implementation of higher vancomycin dose and serum trough concentration per IDSA/ ASHP guidelines. Prospective multicenter, controlled studies evaluating optimal dosing strategies for vancomycin are warranted.
\end{abstract}

\section{Introduction}

Methicillin-resistant Staphylococcus aureus (MRSA) bloodstream infection (BSI) is an important cause of morbidity and mortality with a 30-day all-cause mortality of up to $30 \%$ [1-5]. The current Infectious Diseases Society of America (IDSA) and the American Society of Health-System Pharmacists (ASHP) guidelines recommend a vancomycin loading dose of 25 to $30 \mathrm{mg} / \mathrm{kg}$ and target serum trough concentration of 15 to $20 \mathrm{mg} / \mathrm{L}$ in patients with MRSA BSI [6,7]. The rationale for these recommendations is based on a combination of evidence including outcomes using the ratio of the vancomycin area under the curve (AUC) to minimum inhibitory concentration (MIC) [AUC/MIC], clinical failure in patients with susceptible MRSA strains with higher vancomycin MICs, and inability to achieve target AUC/ MIC in strains with higher MICs from earlier recommended therapy [6-14]. This was particularly important in Detroit, where increased resistance and reduced susceptibility to vancomycin in S. aureus have been a serious concern. The first MRSA cases in the United States were identified in Detroit in the 1980s, which resulted in frequent use of vancomycin [15]. Consequently, the first reported vancomycinintermediate $S$. aureus (VISA) and vancomycin-resistant $S$. aureus (VRSA) emerged from Detroit [16].

There are limited published literatures on the impact of newer dosing strategies on patient outcomes. Therefore, we sought to evaluate mortality difference in MRSA BSI pre and post hospital-wide implementation of higher vancomycin serum trough concentration per IDSA/ASHP guidelines in a large patient cohort over a 9-year period.

\section{Materials and methods}

\section{Study design and patient identification}

This was a retrospective cohort study conducted in a large integrated hospital health system (2238 beds) in Southeast Michigan. All patients who were $\geq 18$ years of age with confirmed MRSA BSI from July 2005 to June 2014 identified via review of microbiology laboratory records were eligible. Patient demographics, comorbid conditions, clinical outcomes, and laboratory data were reviewed. Vancomycin serum trough concentration guidelines, following the publication of IDSA/ASHP recommendations, were implemented in the entire health system since January 2010 by clinical pharmacists as part of the antimicrobial stewardship program. Clinical pharmacists managed vancomycin dosing, monitored and audited levels, and

Correspondence to: Marcus J. Zervos, Division Head, Infectious Diseases, Henry Ford Health System, Professor of Medicine, Wayne State University School of Medicine, Detroit, MI 48202, USA, Tel: +1-313-916-2573; Fax: +1-313-9162993; E-mail: mzervos1@hfhs.org

Key words: bacteremia, mortality, Staphylococcus aureus, trough, vancomycin Received: July 02, 2016; Accepted: July 26, 2016; Published: July 29, 2016 
used established pharmacodynamic dosing models both before and after implementation of the system wide change in dosing. Mortality was evaluated before and after the implementation of the published guideline in the entire health system.

\section{Susceptibility testing}

The initial identification and susceptibility testing of isolates were performed by the clinical microbiology laboratory using VITEK 2 (bioMérieux, Inc., Durham, NC, USA) according to the manufacturer's instructions as set by the Clinical and Laboratory Standards Institute (CLSI) [17]. Vancomycin MICs for all isolates were also determined by Etest method (bioMérieux, Inc.) according to the manufacturer's instructions $[18,19]$.

\section{Statistical analysis}

All continuous data were described using means and standard deviations, while all categorical data were presented as counts and percentages. Univariate two-group tests were used to compare groups: two-group t-tests, analysis of variance, chi-square, and Fisher's exact as appropriate. A Spearman's rank correlation coefficient was used to examine the correlation between mean vancomycin Etest MIC over time. This nonparametric test was chosen due to the ordinal nature of the time variable. The trend in average vancomycin Etest MIC over time was fit using a general linear model. Statistical significance was set at $\mathrm{p}<0.05$. All analyses were performed using SAS 9.4 (SAS Institute Inc., Cary, NC, USA).

\section{Results}

A total of 1,173 patients were evaluated from 2005 to 2014. Overall, the 30 -day all-cause mortality was $16.5 \%$ and did not change after the implementation of the IDSA/ASHP guidelines (Figure 1). The mean MIC, percentage of MRSA isolates with vancomycin MIC $\geq 2.0 \mathrm{mg} / \mathrm{L}$, and mortality per year are shown in Table 1 . Mortality was $16.5 \%$ among patients with high vancomycin MIC ( $\geq 1.5 \mathrm{mg} / \mathrm{L})$ vs. $17.0 \%$ in patients with low vancomycin MIC $(<1.5 \mathrm{mg} / \mathrm{L})$. A summary of mortality over the 9 years in relation to vancomycin susceptibility is shown in Table 1 . There was no statistically significant change in mortality over the 9 years period $(\mathrm{p}=0.193)$. The mean vancomycin MIC over the study period was $1.57 \pm 0.26 \mathrm{mg} / \mathrm{L}$. When evaluating a change in mean vancomycin MIC by Etest method over time, we found a weakly positive correlation coefficient of $0.156(p<0.001)$, when using spearman's correlation. We did not find differences between the pre and post intervention periods $(\mathrm{p}=0.188)$ in MRSA isolates with MIC $\geq 2 \mathrm{mg} / \mathrm{L}$.

\section{Discussion}

This study demonstrated no impact on MRSA BSI mortality rates after the 2010 implementation of IDSA/ASHP guidelines for vancomycin use. Overall mortality $(16.5 \%)$ found in our study was lower than rates published from other institutions [1-5]. We found no increase in vancomycin MICs pre and post intervention that would have an impact on the findings observed. Vancomycin was dosed and monitored by health system pharmacists before and after the guideline change, following established pharmacodynamic modes for dosing, with periodic auditing, and infectious disease physician review of desired targets. Importantly, this study illustrates that despite following dosing guidelines for vancomycin designed to improve outcomes, mortality rates of $S$. aureus bloodstream infections have remained stagnant in a large number of patients before and after implementation of the guidelines hospital wide and over a several year period.

Vancomycin has been routinely used to treat severe MRSA infections since the 1980s; however, despite its use for over 50 years, controlled trials of dosing strategies are not available and optimal dosing remains undefined. In August 2009, target dosing recommendations were altered based on earlier studies demonstrating outcomes of vancomycin therapy utilizing AUC/MIC. Additionally, concern over susceptible strains with higher MICs (MIC creep), failure of therapy due to these organisms, and their inability to achieve targeted AUC/MIC, contributed to the change in recommendations [6-14]. Nevertheless, studies of vancomycin MIC creep have shown variability. Some studies have shown that the MIC creep can be related to methods used for storage of strains, strain selection, single center analysis, year of study, and statistical methods used for analysis [2026]. Conversely, other single-center and large-multicenter studies have not shown increases in MIC even when controlling for strain types [20-24]. When comparing susceptibility testing methods, the MICs reported by the Etest method are higher than those reported by the broth microdilution method $[25,26]$.

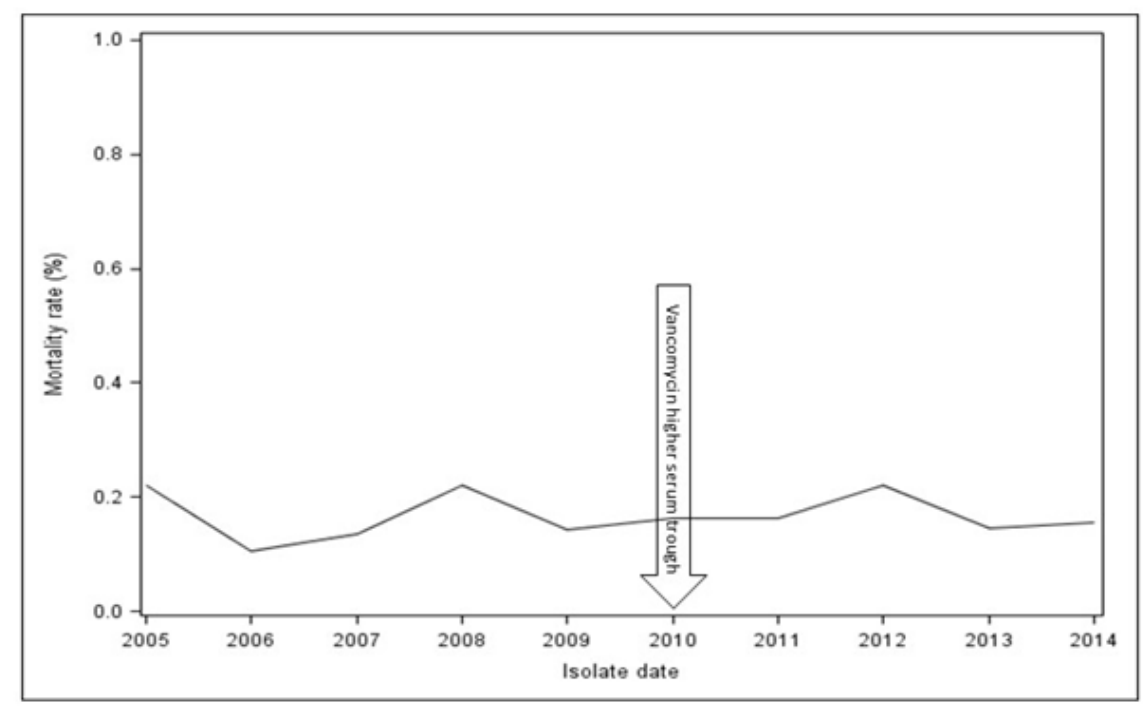

Figure 1. Mortality of MRSA bloodstream infection by year since the implementation of IDSA/ASHP for vancomycin dosing guideline between $2005-2014$. 
Table 1.Vancomycin MICs and 30-day all-cause mortality of MRSA bloodstream infection from 2005-2014.

\begin{tabular}{|c|c|c|c|c|c|c|c|c|c|c|}
\hline & 2005 & 2006 & 2007 & 2008 & 2009 & 2010 & 2011 & 2012 & 2013 & 2014 \\
\hline Patients (n) & 36 & 123 & 119 & 144 & 118 & 137 & 145 & 141 & 147 & 61 \\
\hline Mean MIC mg/L $( \pm$ SD $)$ & $\begin{array}{c}1.58 \\
(0.35)\end{array}$ & $\begin{array}{c}1.59 \\
(0.24)\end{array}$ & $\begin{array}{c}1.45 \\
(0.22)\end{array}$ & $\begin{array}{c}1.53 \\
(0.29)\end{array}$ & $\begin{array}{c}1.66 \\
(0.27)\end{array}$ & $\begin{array}{c}1.51 \\
(0.23)\end{array}$ & $\begin{array}{c}1.51 \\
(0.17)\end{array}$ & $\begin{array}{c}1.59 \\
(0.25)\end{array}$ & $\begin{array}{c}1.68 \\
(0.26)\end{array}$ & $\begin{array}{c}1.66 \\
(0.23)\end{array}$ \\
\hline $\begin{array}{l}\text { Percent }(\%) \text { MRSA with } \\
\text { vancomycin } M I C \geq 2 \mathrm{mg} / \mathrm{L}\end{array}$ & 19.4 & 21.1 & 5 & 6.9 & 31.4 & 8.8 & 6.2 & 19.1 & 36 & 31.1 \\
\hline Mortality (\%) & 22.2 & 10.6 & 13.5 & 22.2 & 14.4 & 16.1 & 16.4 & 22.1 & 14.5 & 15 \\
\hline
\end{tabular}

The rationale for the newer AUC/MIC targets was based on studies that demonstrated susceptible strains with higher MICs were associated with treatment failure [27]. The most recent meta-analysis of 38 studies consisting of 8291 episodes of $S$. aureus bacteremia showed an overall mortality of $26.1 \%$ [27]. Mortality was $26.8 \%$ in patients with high vancomycin MIC ( $\geq 1.5 \mathrm{mg} / \mathrm{L})$ vs. $25.8 \%$ in patients with low vancomycin MIC $(<1.5 \mathrm{mg} / \mathrm{L})$. Additionally, there were no significant differences in risk of death in subgroups with high vs. low vancomycin MIC across different study designs, microbiological susceptibility assays, MIC cutoffs, clinical outcomes, duration of bacteremia, previous vancomycin exposure, and treatment with vancomycin [28]. Furthermore, an optimal vancomycin AUC/MIC has not been established. Although an AUC:MIC $\geq 400$ is the prevailing target, this recommendation is based on data derived from mouse models and uncontrolled trials, which also suggest that the current trough targets are not associated with better outcomes [29-31]. In a study of 200 consecutive patients with MRSA BSI, there was no difference in outcomes comparing patients with vancomycin serum trough levels of $<15 \mathrm{mg} / \mathrm{L}$ or $\geq 15 \mathrm{mg} / \mathrm{L}$ [29]. Finally, the commonly cited AUC/MIC of 400 as the target is based on broth microdilution MICs, which are lower than Etest MICs, and are achievable using lower dosing strategies $[30,31]$. The MIC by Etest method of $1.5-2.0 \mathrm{mg} / \mathrm{L}$ observed in our study is equivalent to $0.5-1.0 \mathrm{mg} / \mathrm{L}$ by broth microdilution, which is within achievable targets.

The goal of achieving a serum vancomycin trough of $15-20 \mathrm{mg} / \mathrm{L}$ for bacteremia must be balanced with toxicity. With earlier serum vancomycin targeted trough of 5-15 $\mathrm{mg} / \mathrm{L}$, the rate of nephrotoxicity was relatively low. However, with higher serum vancomycin targeted trough $(15-20 \mathrm{mg} / \mathrm{L})$, risk of nephrotoxicity is increased, and it can be difficult to maintain levels within the targeted range in patients with fluctuating renal function [31]. Additionally, there is evidence to suggest that there is a significant increase in the rate of nephrotoxicity associated with higher serum vancomycin trough [32]. In a review of 176 patients at 2 teaching hospitals (2008-2011) who received > 7 days of vancomycin and attained high troughs $(15-20 \mathrm{mg} / \mathrm{L}), 14 \%$ experienced nephrotoxicity (creatinine rise of $0.5 \mathrm{mg} / \mathrm{dL}$ or $50 \%$ over baseline on 2 consecutive days) [32]. Risk factors included admission to a general medicine unit, extended duration of treatment ( 7 days), gastrointestinal comorbidity, malignancy, and febrile neutropenia [32]. In a study consisting of 188 intensive care unit patients from 4 centers who received vancomycin for the treatment of pneumonia, nephrotoxicity occurred in $15.4 \%$ of vancomycin-treated patients. Multivariate analysis showed the following variables to be independently associated with nephrotoxicity: initial vancomycin trough levels $\geq 15$ $\mathrm{mg} / \mathrm{L}$ (odds ratio [OR], 5.2 [95\% CI, 1.9-13.9]; $\mathrm{p}=0.001$ ), concomitant aminoglycoside use (OR, 2.67 [95\% CI, 1.09-6.54]; p=0.03), and duration of vancomycin therapy (OR for each additional treatment day, 1.12 [95\% CI, 1.02-1.23]; p 0.02). The incidence of nephrotoxicity increased as a function of the initial vancomycin trough level, rising from $7 \%$ at a trough $<10 \mathrm{mg} / \mathrm{L}$ to $34 \%$ at $>20 \mathrm{mg} / \mathrm{L}(\mathrm{p}=0.001)$. The mean time to nephrotoxicity decreased from 8.8 days at vancomycin trough levels $<15 \mathrm{mg} / \mathrm{L}$ to 7.4 days at $>20 \mathrm{mg} / \mathrm{L}$ (Kaplan-Meier analysis, $\mathrm{p}=0.0003$ ) [33]. Conversely, other studies have shown that vancomycin is minimally nephrotoxic when appropriate dosing is utilized, even among elderly and critically-ill patients with complicated infections [34,35]. However, the collective literature indicates that an exposurenephrotoxicity relationship for vancomycin exists. The probability of a nephrotoxic event increases as a function of trough concentration and duration of therapy. A systematic review of 15 studies, selected from a total of 240 publications from 1996-2012, showed higher vancomycin trough $(\geq 15 \mathrm{mg} / \mathrm{L})$ was associated with increased nephrotoxicity after adjustment for independent risk factors (Odds Ratio 2.67; 95\% CI: 1.95-3.65). An incremental increase in nephrotoxicity was observed with a longer duration of vancomycin. The toxicity was reversible in most cases, with required temporary dialysis in 3\% of toxicity cases, and no mention of end stage renal disease [36].

This study has several limitations, including single center trial, retrospective study design, lack of information on individual dosing of patients and serum trough levels, and uncontrolled confounding variables associated with mortality. Strengths include large numbers of patients with S. aureus bacteremia over a several year period, and dosing carefully done and monitored by experienced hospital pharmacists. Importantly, this study illustrates that despite various advances in medical therapy mortality rates of $S$. aureus bloodstream infections have remained stagnant in a large number of patients. We support the recommendations of individualized vancomycin dosing while taking into consideration the patient's source of infection, underlying medical conditions, particularly renal function, in vitro susceptibility, and previous vancomycin exposure. Therefore, it is incumbent on us to seek better methods of preventing infections and improving dosing strategies and outcomes. Most importantly, our study demonstrates the need for randomized controlled studies of optimal vancomycin dosing strategies that include patient safety as well as outcomes.

\section{Authorship}

All authors mentioned above gave substantial intellectual contribution to this manuscript.

\section{Acknowledgement}

The authors have no specific acknowledgement.

\section{Funding information}

The authors received no specific funding for this work.

\section{Competing interests}

M.J.Z. has received research grants from Pfizer, Cerexa, Cubist, Merck, Tetraphase, and Rempex. K.R. has received research grant from Cerexa, Forest, Actavis, Cubist, Merck, and Theravance. V.H. has received research grant and consultations from Theravance Biopharma, Inc. All other authors have nothing to declare.

\section{References}

1. Hidron AI, Edwards JR, Patel J, Horan TC, Sievert DM, et al. (2008) National 
Healthcare Safety Network Team, Participating National Healthcare Safety Network Facilities. NHSN annual update: antimicrobial-resistant pathogens associated with healthcare-associated infections: annual summary of data reported to the National Healthcare Safety Network at the Centers for Disease Control and Prevention, 20062007. Infect Control Hosp Epidemiol 29: 996-1011.

2. Klevens RM, Morrison MA, Nadle J, Petit S, Gershman K, et al. (2007) Invasive methicillin-resistant Staphylococcus aureus infections in the United States. JAMA 298: 1763-1771. [Crossref]

3. Cosgrove SE, Qi Y, Kaye KS, Harbarth S, Karchmer AW, et al. (2005) The impact of methicillin resistance in Staphylococcus aureus bacteremia on patient outcomes: mortality, length of stay, and hospital charges. Infect Control Hosp Epidemiol 26: 166174.

4. van Hal SJ, Lodise TP, Paterson DL (2012) The clinical significance of vancomycin minimum inhibitory concentration in Staphylococcus aureus infections: a systematic review and meta-analysis. Clin Infect Dis 54: 755-771.

5. Kullar R, Davis SL, Levine DP, Rybak MJ (2011) Impact of vancomycin exposure on outcomes in patients with methicillin-resistant Staphylococcus aureus bacteremia: support for consensus guidelines suggested targets. Clin Infect Dis 52: 975-981.

6. Rybak M, Lomaestro B, Rotschafer JC, Moellering R Jr, Craig W, et al. (2009) Therapeutic monitoring of vancomycin in adult patients: a consensus review of the American Society of Health-System Pharmacists, the Infectious Diseases Society of America, and the Society of Infectious Diseases Pharmacists. Am J Health Syst Pharm 66: 82-98.

7. Liu C, Bayer A, Cosgrove SE, Daum RS, Fridkin SK, et al. (2011) Clinical practice guidelines by the infectious diseases society of america for the treatment of methicillinresistant Staphylococcus aureus infections in adults and children: executive summary. Clin Infect Dis 52: 285-292.

8. Patel N, Pai MP, Rodvold KA, Lomaestro B, Drusano GL, et al. (2011) Vancomycin: we can't get there from here. Clin Infect Dis 52: 969-974. [Crossref]

9. Rhee KY, Gardiner DF, Charles M (2005) Decreasing in vitro susceptibility of clinical Staphylococcus aureus isolates to vancomycin at the New York Hospital: quantitative testing redux. Clin Infect Dis 40: 1705-1706. [Crossref]

10. Steinkraus G, White R, Friedrich L (2007) Vancomycin MIC creep in non-vancomycinintermediate Staphylococcus aureus (VISA), vancomycin-susceptible clinical methicillin-resistant S. aureus (MRSA) blood isolates from 2001-05. J Antimicrob Chemother 60: 788-794.

11. Wang G, Hindler JF, Ward KW, Bruckner DA (2006) Increased vancomycin MICs for Staphylococcus aureus clinical isolates from a university hospital during a 5-year period. J Clin Microbiol 44: 3883-3886. [Crossref]

12. Awad SS, Elhabash SI, Lee L, Farrow B, Berger DH (2007) Increasing incidence of methicillin-resistant Staphylococcus aureus skin and soft-tissue infections: reconsideration of empiric antimicrobial therapy. Am J Surg 194: 606-610. [Crossref]

13. Yeh YC1, Yeh KM, Lin TY, Chiu SK, Yang YS, et al. (2012) Impact of vancomycin MIC creep on patients with methicillin-resistant Staphylococcus aureus bacteremia. $J$ Microbiol Immunol Infect 45: 214-220. [Crossref]

14. Dhand A, Sakoulas G (2012) Reduced vancomycin susceptibility among clinical Staphylococcus aureus isolates ('the MIC Creep'): implications for therapy. F1000 Med Rep 4: 4

15. Crane LR, Levine DP, Zervos MJ, Cummings G (1986) Bacteremia in narcotic addicts at the Detroit Medical Center. I. Microbiology, epidemiology, risk factors, and empiric therapy. Rev Infect Dis 8: 364-373.

16. Sievert DM1, Rudrik JT, Patel JB, McDonald LC, Wilkins MJ, et al. (2008) Vancomycin-resistant Staphylococcus aureus in the United States, 2002-2006. Clin Infect Dis 46: 668-674. [Crossref]

17. Sader HS1, Flamm RK, Jones RN (2013) Antimicrobial activity of daptomycin tested against Gram-positive pathogens collected in Europe, Latin America, and selected countries in the Asia-Pacific Region (2011). Diagn Microbiol Infect Dis 75: 417-422. [Crossref]

18. Clinical and Laboratory Standards Institute (CLSI) (2006) Methods for Antimicrobial Susceptibility Tests for Bacteria That Grow Aerobically- Ninth Edition: Approved Standard 32: 1-68.

19. Clinical Laboratory Standards Institute (CLSI) (2011) Performance Standards for Antimicrobial Susceptibility Testing. Twenty-First Informational Supplement 30: $1-165$.

20. Shoyinka A, Moreno D, Arshad S, Perri MB, Donabedian SM, et al. (2014) Evaluation of in vitro susceptibility trends to vancomycin and daptomycin by strain type of Staphylococcus aureus causing bloodstream infections. J Glob Antimicrob Resist 2: 280-285.

21. Alós JI, García-Cañas A, García-Hierro P, Rodríguez-Salvanés F (2008) Vancomycin MICs did not creep in Staphylococcus aureus isolates from 2002 to 2006 in a setting with low vancomycin usage. J Antimicrob Chemother 62: 773-775. [Crossref]

22. Reynolds R, Hope R, Warner M, MacGowan AP, Livermore DM, et al. (2012) Lack of upward creep of glycopeptide MICs for methicillin-resistant Staphylococcus aureus (MRSA) isolated in the UK and Ireland 2001-07. J Antimicrob Chemother 67: 2912 2918. [Crossref]

23. Sader HS, Fey PD, Limaye AP, Madinger N, Pankey G, et al. (2009) Evaluation of vancomycin and daptomycin potency trends (MIC creep) against methicillin-resistant Staphylococcus aureus isolates collected in nine U.S. medical centers from 2002 to 2006. Antimicrob Agents Chemother 53: 4127-4132.

24. Edwards B, Milne K, Lawes T, Cook I, Robb A, et al. (2012) Is vancomycin MIC "creep" method dependent? Analysis of methicillin-resistant Staphylococcus aureus susceptibility trends in blood isolates from North East Scotland from 2006 to $2010 . J$ Clin Microbiol 50: 318-325.

25. van Hal SJ, Wehrhahn MC, Barbagiannakos T, Mercer J, Chen D, et al. (2011) Performance of various testing methodologies for detection of heteroresistant vancomycin-intermediate Staphylococcus aureus in bloodstream isolates. J Clin Microbiol 49: 1489-1494.

26. Prakash V, Lewis JS 2nd, Jorgensen JH (2008) Vancomycin MICs for methicillinresistant Staphylococcus aureus isolates differ based upon the susceptibility test method used. Antimicrob Agents Chemother 52: 4528. [Crossref]

27. Kalil AC, Van Schooneveld TC, Fey PD, Rupp ME (2014) Association between vancomycin minimum inhibitory concentration and mortality among patients with Staphylococcus aureus bloodstream infections: a systematic review and metaanalysis. JAMA 312: 1552-1564. [Crossref]

28. Holmes NE, Turnidge JD, Munckhof WJ, Robinson JO, Korman TM, et al. (2011) Antibiotic choice may not explain poorer outcomes in patients with Staphylococcus aureus bacteremia and high vancomycin minimum inhibitory concentrations. $J$ Infect Dis 204: 340-347. [Crossref]

29. Arshad S, Shoyinka A, Chen A, Jacobsen G, Zervos M (2012) Evaluation of vancomycin serum trough concentrations and outcomes in meticillin-resistant Staphylococcus aureus bacteraemia. Int $J$ Antimicrob Agents 40: 474-475.

30. Moise PA, Forrest A, Bhavnani SM, Birmingham MC, Schentag JJ (2000) Area under the inhibitory curve and a pneumonia scoring system for predicting outcomes of vancomycin therapy for respiratory infections by Staphylococcus aureus. Am J Health Syst Pharm 2: S4-S9.

31. Moise-Broder PA, Forrest A, Birmingham MC, Schentag JJ (2004) Pharmacodynamics of vancomycin and other antimicrobials in patients with Staphylococcus aureus lower respiratory tract infections. Clin Pharmacokinet 43: 925-942.

32. Contreiras C, Legal M, Lau TT, Thalakada R, Shalansky S, et al. (2014) Identification of risk factors for nephrotoxicity in patients receiving extended-duration, high-trough vancomycin therapy. Can J Hosp Pharm 67: 126-132. [Crossref]

33. Cano EL, Haque NZ, Welch VL, Cely CM, Peyrani P, et al. (2012) Incidence of nephrotoxicity and association with vancomycin use in intensive care unit patients with pneumonia: retrospective analysis of the IMPACT-HAP Database. Clin Ther 34: 149 157. [Crossref]

34. Davies SW, Guidry CA, Petroze RT, Hranjec T, Sawyer RG (2013) Vancomycin and nephrotoxicity: just another myth? J Trauma Acute Care Surg 75: 830-835. [Crossref]

35. Carreno JJ, Jaworski A, Kenney RM, Davis SL (2013) Comparative Incidence of Nephrotoxicity by Age Group among Adult Patients Receiving Vancomycin. Infec Dis Ther 2: 201-208.

36. van Hal SJ, Paterson DL, Lodise TP (2013) Systematic review and meta-analysis of vancomycin-induced nephrotoxicity associated with dosing schedules that maintain troughs between 15 and 20 milligrams per liter. Antimicrob Agents Chemother 57: 734-744.

Copyright: (C2016 Bardossy AC. This is an open-access article distributed under the terms of the Creative Commons Attribution License, which permits unrestricted use, distribution, and reproduction in any medium, provided the original author and source are credited. 